\title{
DÜBLIN
}

Technological University Dublin

ARROW@TU Dublin

\section{Effects of Mutations in the Helix G Region of Horseradish Peroxidase}

\author{
Barry Ryan \\ Technological University Dublin, barry.ryan@tudublin.ie \\ Ciarán O'Fágáin \\ Dublin City University
}

Follow this and additional works at: https://arrow.tudublin.ie/schfsehart

Part of the Life Sciences Commons

\section{Recommended Citation}

Ryan, B. \& Ó Fágáin, C. (2007). Effects of mutations in the Helix G region of Horseradish Peroxidase.

Biochemie, vol. 89,(8), pp.1029-1032. doi:10.1016/j.biochi.2008.05.008

This Article is brought to you for free and open access by the School of Food Science and Environmental Health at ARROW@TU Dublin. It has been accepted for inclusion in Articles by an authorized administrator of ARROW@TU

Dublin. For more information, please contact

arrow.admin@tudublin.ie, aisling.coyne@tudublin.ie, gerard.connolly@tudublin.ie.

Funder: Embark Initiative and Dublin City University

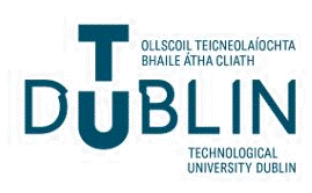




\title{
Effects of mutations in the Helix G region of Horseradish Peroxidase
}

\author{
Barry J. Ryan and Ciarán Ó’Fágáin* \\ School of Biotechnology and National Centre for Sensors Research, Dublin City University, \\ Dublin 9, Ireland.
}

Running Title: Mutations in the horseradish peroxidase helix G region.

\footnotetext{
* corresponding author: Ciarán Ó'Fágáin (Ciaran.Fagan@ dcu.ie).

Phone: $+353-1-7005288$

Fax: $\quad+353-1-7005412$

www.dcu.ie/biotechnology/index.shtml
}

Key Words: Recombinant, Horseradish Peroxidase, Site-Specific Mutagenesis, Protein Stabilisation. 


\section{Summary.}

Horseradish Peroxidase (HRP) has long attracted intense research interest and is used in many biotechnological fields, including diagnostics, biosensors and biocatalysis. Enhancement of HRP catalytic activity and/or stability would further increase its usefulness. Based on prior art, we substituted solvent-exposed lysine and glutamic acid residues near the proximal helix G (Lys 232, 241; Glu 238, 239) and between helices F and F' (Lys 174). Three single mutants (K232N, K232F, K241N) demonstrated increased stabilities against heat (up to two-fold) and solvents (up to four-fold). Stability gains are likely due to improved hydrogen bonding and space-fill characteristics introduced by the relevant substitution. Two double mutants showed stability gains but most double mutations were non-additive and non-synergistic. Substitutions of Lys 174 or Glu 238 were destabilising. Unexpectedly, notable alterations in steady-state $V_{\mathrm{m}} / E$ values occurred with reducing substrate ABTS (2,2'-azino-bis(3ethylbenzthiazoline-6-sulfonic acid)), despite the distance of the mutated positions from the active site.

Abbreviations: ABTS, 2,2'-azino-bis(3-ethylbenzthiazoline-6-sulfonic acid); $\mathrm{C}_{50}$, v/v solvent concentration where $50 \%$ catalytic activity remains; $\delta$-ALA, delta aminolevulinic Acid; DMF, dimethylformamide; DMSO, dimethylsulfoxide; EGNHS, ethylene glycol bis-succinimidyl succinate; HRP, horseradish peroxidase isoenzyme $\mathrm{C}$; $\mathrm{GnCl}$, guanidine hydrochloride; $\mathrm{LB}$, Luria-Bertani medium; $\mathrm{MeOH}$, methanol; PA, phthalic anhydride; rHRP, recombinant horseradish peroxidase isoenzyme C; RZ, reinheitszahl (purity number; $A_{403} / A_{280}$ ); $t_{1 / 2}$, halflife; TMB, 3,3',5'5-tetramethyl benzidine; v/v, volume per volume; w/v, weight per volume. 


\section{Introduction.}

The most widely studied peroxidase is isoform $\mathrm{C}$ from horseradish roots (Armoracia rusticana; HRP), due mainly to its many uses in biotechnology [1]. Improvement of HRP's catalytic properties and/or stability would further extend its range of applications. Initial HRP mutagenesis studies set out to identify the critical residues involved in HRP catalysis [1]. There are a few reports of serendipitously altered catalytic properties, such as peroxygenase activity ([2]; His $42 \rightarrow$ Glu), thioanisole sulfoxidation ([3]; His42 $\rightarrow$ Ala/Phe41 $\rightarrow$ His), or both ([4]; Phe $41 \rightarrow$ Leu). There are also reports of altered kinetic properties arising from single substitutions: for example, an increased $V_{\mathrm{m}} / E$ ratio for ABTS resulted from a single Ser35 $\rightarrow$ Lys substitution [5], whilst a Asn70 $\rightarrow$ Asp substitution led to decreased guaiacol oxidation rates [6]. A recent paper describes HRP active site variants with enhanced enantioselectivity for L- or D-tyrosinol identified by yeast cell surface display [7].

The stability of HRP has a crucial bearing on its applicability and is affected by a number of variables including temperature [8,9,10], calcium content [10], glycosylation [11], pressure [12], irradiation [13] and $\mathrm{pH}[9,14]$, along with additional factors such as urea $[15,16]$, guanidinium chloride [15,17] and various solvents including dimethyl sulfoxide [18] and ionic liquids [19]. Directed evolution has been used to alter rHRP thermal stability [20] but we have found no reports of HRP stability manipulation via rational, site-directed mutagenesis.

Among non-genetic methods, chemical reactions such as crosslinking $[21,22,23,24,25]$ and surface modification [26, 27, 28, 29] have successfully increased HRP's stability. Notably, these beneficial modifications target HRP's solvent-exposed lysine residues. Thermostabilisation of HRP by treatment with the bifunctional compound EGNHS involves only three of HRP's six lysine residues (crosslinking of Lys232 with Lys241; modification of Lys 174); the others (Lys 65, 84 and 149) are scarcely modified at all under mild conditions 
[23]. Examination of the HRP crystal structure indicates that Lys 65, 84 and 149 are not easily accessible for reaction due to restricted exposure to solvent, participation in salt pairing and steric hindrance by a glycan moiety [23]. Clearly, the lysines at positions 174, 232 and 241 influence HRP stability and thus provide targets for possibly stabilising rational mutations. We performed amino acid substitutions at these positions to mimic the molecular effects of chemical modification. Negatively charged glutamic acid residues (E238 and E239, situated between the crosslinkable $\mathrm{K} 232$ and K241) were also investigated, together with double lysine mutants. Some of the mutations yielded modest stability gains. Unexpectedly, notable alterations in steady-state kinetic values occurred with ABTS as reducing substrate, despite the distance of the mutated sites from the active site.

\section{Materials and Methods.}

Materials. The HRP gene was a generous gift from Prof. Frances H. Arnold (Caltech, CA, USA). The pQE60 vector was purchased from Qiagen (Valencia, CA); XL 10 Gold cells and QuickChange $^{\mathrm{TM}}$ Mutagenesis Kit were purchased from Stratagene (La Jolla, CA). All reagents were purchased from Sigma Aldrich and were of analytical grade or higher.

Cloning. Based on refs. [30, 31], the HRP gene was directionally cloned into the pQE60 vector as a fusion with the $\mathrm{N}$-terminal pectate lyase (PelB) leader sequence [32] and a Cterminal hexa-histidine purification tag, to generate the plasmid pBR_I. Initially, the PelB leader was cloned via a Nco I - BamH I double restriction. This introduced a novel Not I site 5' to the existing BamH I site in the modified pQE60 vector. The HRP gene was then Not I Bgl II directionally cloned into a Not I - BamH I restricted PelB-modified pQE60 vector. This

cloning strategy incorporated the poly-His tag, present in the pQE60 vector, at the C-terminus of HRP (see Figure 1). 
Bacterial Strains and Plasmids. E.coli XL 10 Gold was used as host strain to express the HRP protein. The plasmid (pBR_I), carrying the HRP gene and coding for the HRP fusion protein, was used for expression and site directed mutagenesis.

Recombinant DNA Techniques. All DNA manipulations were carried out by standard techniques [33]. Site directed mutagenesis was carried out as described by Wang and Malcom [34] utilising the QuickChange ${ }^{\mathrm{TM}}$ method. Mutational primers were supplied by MWGBiotech (Germany). Mutants were confirmed by commercial di-deoxy sequencing (Fusion Antibodies, Belfast, Northern Ireland).

Expression and Purification. A single cell transformed with pBR_I (or mutant derivative) was grown in $10 \mathrm{~mL}$ LB medium containing $100 \mu \mathrm{g} / \mathrm{mL}$ ampicillin and $2 \% \mathrm{w} / \mathrm{v}$ glucose until the $\mathrm{OD}_{600 \mathrm{~nm}}$ reached 0.4 ; the cells were removed via centrifugation at $2,000 \times g$ for $5 \mathrm{~min}$ and resuspended in fresh LB $(500 \mathrm{~mL})$ supplemented with $100 \mu \mathrm{g} / \mathrm{mL}$ ampicillin, $1 \mathrm{mM} \delta$-ALA and $2 \mathrm{mM} \mathrm{CaCl} 2$. The cells were then allowed to grow at $30^{\circ} \mathrm{C}, 220 \mathrm{rpm}$ for $16 \mathrm{~h}$. Following overnight expression, the cells were centrifuged at $2,000 \times g$ for $5 \mathrm{~min}$ and the supernatant was treated with $50 \% \mathrm{w} / \mathrm{v}$ ammonium sulphate for $2 \mathrm{~h}$ at room temperature. The cells were periplasmically lysed [35] and the periplasmic contents were similarly treated with $50 \% \mathrm{w} / \mathrm{v}$ (with respect to the initial supernatant volume) ammonium sulphate. Proteins precipitated by ammonium sulphate from both the culture supernatant and the periplasmic preparation were collected via centrifugation, resuspended in $50 \mathrm{mM}$ phosphate buffer $\mathrm{pH} 7.5$, pooled and dialysed versus the same buffer overnight at $4^{\circ} \mathrm{C}$. Sodium chloride $(1 \mathrm{M})$ and $\mathrm{GnCl}(200 \mathrm{mM})$ were added to the dialysed fractions (10 $\mathrm{mL}$ total volume), and these latter were purified via nickel affinity chromatography at room temperature. Sodium acetate $(25 \mathrm{mM}, \mathrm{pH} 4.5)$ was 
utilised to elute the bound HRP. The eluted HRP was again dialysed versus 50mM phosphate buffer pH 7.5 overnight at $4^{\circ} \mathrm{C}$, after which the protein was concentrated (Amicon-Plus 20 concentrator tubes; $2 \mathrm{~mL}$ final volume), filter sterilised and stored at $4^{\circ} \mathrm{C}$. These procedures led to typical expression values of $0.086 \mathrm{mg}$ HRP per litre of culture medium. Purified HRP (specific activity $0.58 \mu \mathrm{mol} \cdot \mathrm{min}^{-1} \cdot \mathrm{mg}^{-1}, \mathrm{RZ}$ value 1.1 ) gave a single band on a $12 \%$ polyacrylamide gel (not shown).

\section{Enzyme Assay and Characterisation.}

The microtitre ABTS assay, modified from ref. [36], comprised $2.5 \mu \mathrm{L}$ of $100 \mathrm{mM} \mathrm{H}_{2} \mathrm{O}_{2}$, $222.5 \mu \mathrm{L}$ of the desired ABTS concentration $(0.1$ to $1.0 \mathrm{mM})$ and $25 \mu \mathrm{L}$ of $\mathrm{rHRP}(30 \mathrm{pM})$ in each well. The microplate was shaken as the initiating enzyme was added and the absorbance at $405 \mathrm{~nm}$ was recorded every minute for $20 \mathrm{~min}$. The change in absorbance per minute $(\Delta \mathrm{A} / \mathrm{min})$ was calculated for each substrate concentration. The Enzfitter (Biosoft, Cambridge, UK) programme fitted the data generated to a Michaelis-Menten equation and calculated apparent kinetic values.

Thermal stabilities of recombinant HRP and mutant variants were determined as for plant HRP [25,27] using $50 \mathrm{mM}$ phosphate buffer, $\mathrm{pH} 7.0$, in which renaturation of unfolded HRP does not occur. For $t_{1 / 2}$ estimation, a single HRP stock solution (room temperature) was plunged into a $50^{\circ} \mathrm{C}$ waterbath. Samples were removed onto ice every minute up to $10 \mathrm{~min}$ and their remaining activities determined under optimal conditions upon re-warming to room temperature. This procedure allows estimation of the first-order rate constant $k$ (Enzfitter programme; Biosoft, Cambridge, UK) and, hence, the apparent half-life, $t_{1 / 2}$. In addition, a thermal profile was generated by placing a single HRP stock solution at progressively increasing temperatures for $10 \mathrm{~min}$, at which time aliquots were withdrawn, chilled on ice and their remaining activities assayed as above (see Figure 2). 
The $\mathrm{C}_{50}$ value (\% v/v solvent concentration which maintains $50 \%$ of aqueous activity, see Table 1) was utilised to compare organo-tolerance among the mutants. Briefly, rHRP was prepared to a concentration of $100 \mu \mathrm{g} \cdot \mathrm{mL}^{-1}$ in $50 \mathrm{mM}$ phosphate buffer, $\mathrm{pH}$ 7.0. Reaction mixtures were set up with $10 \%$ increasing volumes of the solvent of interest in $50 \mathrm{mM}$ phosphate buffer, $\mathrm{pH} 7.0$, in the range $0 \%-90 \%$ v/v solvent. Samples were exposed to the relevant solvent concentration for 2 hours at $25{ }^{\circ} \mathrm{C}$ in a temperature-controlled waterbath followed by measurement of residual activity under optimal conditions. In all stability analyses, residual catalytic activity was assayed using TMB as the reducing substrate [25].

\section{Results.}

Over a 10-min incubation period, the half-inactivation temperatures for our wild type recombinant and plant $\mathrm{HRP}$ were $50^{\circ} \mathrm{C}$ and $55^{\circ} \mathrm{C}$ respectively. The greater stability of the plant enzyme is probably due to glycosylation [11]. Thermal profiles of His-tagged and non His-tagged wildtype recombinant HRP closely resembled each other (data not shown). For convenience, the His-tagged form was used subsequently. Thermal inactivations (over time at constant $50^{\circ} \mathrm{C}$; Table 1) and thermal profiles (Fig. 2) were each carried out at constant protein concentration $\left(0.1 \mathrm{mg} \cdot \mathrm{ml}^{-1}\right)$ to control for possible concentration effects on stability. Except for K241E and K241N, rHRP thermoinactivations fitted satisfactorily to a first-order decay $\left(\mathrm{r}^{2}\right.$ $>0.91$ ) over the first $10 \mathrm{~min}$, allowing estimation of a first-order $k$-value and, hence, an apparent half-life $\left(t_{1 / 2}\right)$ at $50^{\circ} \mathrm{C}$. (Deviations did occur at longer times, however; HRP thermal denaturation tends towards a 1.5 -order process [37].)

Two single mutants (K232N and $\mathrm{K} 232 \mathrm{~F}$ ) exhibited longer $t_{1 / 2}$ (by $80 \%$ and $93 \%$, respectively), as did all of the double mutants (K232Q/K241Q, 23\%; K232N/K241N, 56\%; and K232F/K241N, 120\%; see Table 1). Neither K241E nor K241N fitted a first- nor a second- 
order decay model; however, $30 \%$ of the starting K241N activity remained after 30 min at the higher temperature of $70^{\circ} \mathrm{C}$; this was much greater than for wildtype.

In the thermal profile (10 min incubations; see Fig. 2) K241N displayed a plateau of stability between $55^{\circ} \mathrm{C}$ and $70^{\circ} \mathrm{C}$, suggesting the existence of an intermediate, partially active isoform. Six out of eleven single Lys mutants, however, displayed shorter $t_{1 / 2}$ than wildtype, with the largest decrease (28\%) noted for K232A (see Table 1).

The effects of water miscible organic solvents, chosen for their denaturation capacities (Methanol, low; and DMF, medium; [38]), and on previous plant peroxidase studies (DMSO; [18]), were also analysed. Wild type rHRP and all mutants underwent inactivation as the percentage volume of organic solvent increased. Three single mutants (K174N, K232N, $\mathrm{K} 232 \mathrm{~F})$, however, displayed increased tolerance in DMSO, one mutant (K232F) in $\mathrm{MeOH}$ and four in DMF (K232N, K232A, K232F, K241A). All double mutants exhibited similar or increased $(\mathrm{K} 232 \mathrm{~F} / \mathrm{K} 241 \mathrm{~N})$ tolerances in the solvents examined (except K232Q/K241Q in DMF).

The large, four-ring [39] reducing substrate ABTS gives steady-state kinetics, permitting estimation of the apparent kinetic parameters $V_{\mathrm{m}} / E$ and $\mathrm{K}_{\mathrm{m}}[36,40]$. Our $\mathrm{rHRP}$ gave $V_{\mathrm{m}} / E$ and apparent $\mathrm{K}_{\mathrm{m}}$ values of $482 \mathrm{~s}^{-1}$ for $V_{\mathrm{m}} / E$ and $0.093 \mathrm{mM}$ for $K_{\mathrm{m}}^{\prime}$ (Table I). These compare with literature values of $670 \mathrm{~s}^{-1}, 0.18 \mathrm{mM}$ and $810 \mathrm{~s}^{-1}, 0.27 \mathrm{mM}$ for recombinant and plant HRP (both RZ >3) respectively [36], obtained under similar conditions.

Single mutant $\mathrm{K}_{\mathrm{m}}$ values varied between $+/-80 \%$ of the wildtype; however, the majority displayed an increased $\mathrm{K}_{\mathrm{m}}$ of $25 \%$ to $50 \%$. Double mutants displayed similar $\mathrm{K}_{\mathrm{m}}^{\prime}$ values, except for $\mathrm{K} 232 \mathrm{~N} / \mathrm{K} 241 \mathrm{~N}$, which yielded a 2-fold increase over wildtype. Effects on $V_{\mathrm{m}} / E$ are greater, with significant increases noted for both single (5-fold, K241A; 3.8-fold, K232N; and 2.7-fold, E238Q) and double mutants (3.2-fold, K232Q/K241Q; and 2-fold, K232F/K241N; 
see Table 1). Neutralisation of Glu 238 and 239 negative charges enhanced HRP's reactivity with ABTS through increased $V_{\mathrm{m}} / E$ and decreased $\mathrm{K}_{\mathrm{m}}^{\prime}$. (Table 1 ).

\section{Discussion.}

Most stabilising chemical modifications of HRP target the solvent-exposed lysines 174, 232 and 241 [23]. O'Brien et al. [23] demonstrated that only these three (of six) lysine residues are modified under mild conditions by the crosslinker EGNHS. Clearly, these three lysines influence HRP stability [24, 25], yet exhaustive modification of all six lysines leads to decreased stability [41]. If other residues (e.g. His) are targeted, the results are stabilityneutral or -negative $[25,42]$. Chemical modification can also influence catalytic properties: marginally improved catalytic activity, but little $\mathrm{K}_{\mathrm{m}}$ alteration, occurred with ABTS and other substrates following chemical modification of HRP with PA and EGNHS [27].

Here, HRP's modifiable lysines 174, 232 and 241 were substituted with the aim of improving thermal stability. Previous stabilizing modifications had neutralized [29] or reversed [27] the positive charges of lysines. Hence, replacement amino acids included neutralising (Asn) or charge-reversing (Glu) amino acids at each of the three positions, or small/ large amino acids (Ala/ Phe) to alter the space-filling characteristics.

All positions mutated yielded altered $t_{1 / 2}$ values compared with wildtype (Table 1 ). The effects differ with position and substitution; for example, all three mutants of Lys 174, located between helices F and F', display decreased stability ( 20\%; as deduced from the $\left.t_{1 / 2}\right)$. DeepView [43] molecular modelling of this region of the HRP structure (PDB Accession number 1ATJ; [44]) indicates a putative hydrogen bond between K174 and the propionate group bound to pyrrole III (Fischer numbering system [40]) of HRP's ferriprotoporphyrin IX. Substitution of K174 will disrupt this interaction, perhaps loosening the heme moiety. It appears that Lys 174 is important for HRP stability and that its replacement is detrimental. 
Previous chemical modifications, at 28\% [23], were not exhaustive and merely altered the properties of this residue without actually replacing it.

Lys-to-Asn mutations at positions 232 and 241, or Lys-to-Phe at position 232, generated proteins with greater thermostability than wildtype but Ala is detabilising in either position. DeepView modelling of 1ATJ suggests that K232 hydrogen bonds with Asp 230 and Asn 236. The K232N substitution may improve hydrogen bonding (introduction of an additional Asn 231 bond; see Fig. 3), whilst maintaining pre-existing bonds. Also, since Asp 230 bonds directly to the proximal $\mathrm{Ca}^{2+}$, better hydrogen bonding by the substituting Asn may strengthen the $\mathrm{Ca}^{2+}$ binding pocket [45]. Consolidation of this pocket may delay loss of the proximal calcium ion, which is known to play a pivotal role in HRP stabilisation [46]. This is reflected in the increased thermal (1.8-fold, Table 1) and solvent (DMF, 3.4-fold; Table 1) stabilities of K232N. A space-filling substitution, K232F, replaces some hydrogen bonds in this region, yet still yields increased thermal (2-fold) and solvent stabilities, perhaps as a result of compensatory packing. Molecular dynamics simulations indicate that additional burial of hydrophobic residues occurs in HRP following chemical modification [47]. A similar type of rearrangement may arise from the stabilizing $\mathrm{K} 232 \mathrm{~F}$ substitution. The importance of hydrogen bonding and space-fill within this region is also highlighted by comparison of K232A and K232N substitutions. Both mutations remove the lysine's positive charge; however, the smaller, non hydrogen-bonding Ala is the most destabilizing substitution (28\%). K241 hydrogen bonds with Asn236 (Deep View). Removal of this H-bond (by Ala or Phe substitution) decreases stability. Substitution by Asn (in the thermostabilized K241N) likely promotes some favourable alternative H-bonding arrangement, despite reducing the side chain length by one $-\mathrm{CH}_{2}$ - and removing the positive charge of the wild type Lys.

Molecular dynamic simulations indicate that chemical modification of Lys 232 and 241 in the Helix G region affects stability by reducing flexibility (i) around the heme moiety and (ii) in 
the overall protein backbone [47]. Previously, HRP's improved stability following carboxylic acid anhydride modification was attributed to altered conformational arrangement and restricted mobility, as indicated by circular dichroism [41]. Our stabilising substitutions at these positions may enhance hydrogen bonding and, as a result, reduce molecular flexibility. Differing effects arise from removal of the negative charge of each of the paired Glu residues at positions 238 and 239. Neutralisation of Glu238 with the similarly sized Gln causes a $60 \%$ decrease in thermal stability, whereas Glu239 $\rightarrow$ Asn is similar to wildtype, indicating that the negative charge at position 239 does not influence stability.

Double mutants (K232Q/K241Q, K23N2/K241N and K232F/K241N) revealed that the stabilising effects of the single mutations are not additive. Although an increase in thermostability is noted for the double Asn mutant, it is not as stable as the individual K232N ( $21 \%$ increase in $t_{1 / 2}$ for $\mathrm{K} 232 \mathrm{~N} / 241 \mathrm{~N}$ as compared with $70 \%$ for $\mathrm{K} 232 \mathrm{~N}$ ). Although the $\mathrm{K} 241 \mathrm{~N}$ single mutant does not undergo a first-order thermal inactivation at $50^{\circ} \mathrm{C}$, double mutants incorporating this substitution (K232N/K241N and K232F/K241N) give satisfactory first-order fits. K241N displays an anomalous thermal profile (Fig. 2; 10 min incubations at increasing temperatures) where, following a steep activity decline above $40^{\circ} \mathrm{C}$, roughly $40 \%$ of initial activity persists in the range $55-70^{\circ} \mathrm{C}$. This suggests that a partially-active intermediate form of $\mathrm{K} 241 \mathrm{~N}$ exists between $55-70^{\circ} \mathrm{C}$, perhaps related to the loss of a calcium ion. Position 241 is not far from HRP's proximal calcium. Approx. 50\% of native activity remained following removal of one of HRP's two calciums and " $\mathrm{Ca}^{2+}$ ions are necessary for optimum catalysis but are not essential for low levels of activity" [45]. Pina et al. [8] noted folding intermediate(s) in a calorimetric study of HRP unfolding. (We have not yet studied unfolding of the polypeptide by physical techniques due to the low expression levels achievable with E. coli cells.) With the double mutants, we believe that the additional stabilizing replacements at position $232(\mathrm{~K} 232 \mathrm{~N}$ and K232F) exert a predominant effect 
during the period of thermal inactivation at $50^{\circ} \mathrm{C}$, masking the anomalous behaviour of K241N and leading to an apparently first-order decay.

The solvent DMSO is known to induce loss of plant HRP secondary structure and of the heme prosthetic group at high concentrations ( $>80 \% \mathrm{v} / \mathrm{v}$ [18]). Recombinant HRP (and mutant derivatives) displayed a much lower DMSO tolerance than plant HRP, suggesting a stabilising role for glycosylation. DMF-induced denaturation of HRP has been attributed to alteration of the enzyme conformation and reduction of the water content close to the protein $[29,48]$. The replacement of a positively-charged Lys with a neutral Asn or negativelycharged Glu may allow the protein to retain bound water molecules in higher concentrations of organic solvent [49], resulting in improved solvent tolerance (e.g. K232N DMF tolerance). This compares well with previous chemical modification results, namely the alteration of the charge on HRP lysines $232 / 241$ by succinimides resulting in significantly improved DMF stability [24]. Only two mutants (K232F and K232F/K241N), however, displayed increased tolerance across the three solvents tested. As with thermal stability, double mutations are nonsynergistic and non-additive in relation to solvent stability; individually, $\mathrm{K} 232 \mathrm{~N}$ and $\mathrm{K} 241 \mathrm{~N}$ yield 3.4- and 1.3-fold gains, whereas their combination $(\mathrm{K} 232 \mathrm{~N} / \mathrm{K} 241 \mathrm{~N})$ results in a modest 1.4-fold gain.

The present thermal stability gains at $50^{\circ} \mathrm{C}$ are less than those obtained from chemical modification of HRP, where previous reports (albeit under different conditions from those used here) have outlined improvements up to 23 -fold at $72.5^{\circ} \mathrm{C}$ from bis-succinimide modification [25], a 5-fold enhancement at $65^{\circ} \mathrm{C}$ with acetic acid $\mathrm{N}$-hydroxysuccinimide ester modification [29] and a 4-fold increase with phthalic anhydride [27]. Attachment of methoxypoly(ethylene glycol) enhanced stability by up to 59-fold [50] while reactions with anhydrides of mono- and dicarboxylic acids and picryl sulfonic acid were "substantially stabilizing" [41]. It is perplexing that mutations at these sites, which clearly have a bearing on 
HRP stability, should yield such modest stability gains. Nonetheless, the present work mirrors previous studies in which amino acid substitutions, not necessarily located in the protein core, can modestly affect the thermostability of recombinant proteins $[51,52,53,54]$. An unanticipated benefit of mutations $\mathrm{K} 232 \mathrm{~N}$ and K241F was greatly-enhanced resistance to inactivation by excess hydrogen peroxide [55]

Most mutants had altered $\mathrm{K}_{\mathrm{m}}$ and $V_{\mathrm{m}} / E$ values for ABTS in aqueous buffer. (Measurement of individual rate constants by an alternative steady-state methodology [56] has yet to be undertaken.) The residues analysed in this study are distant from the HRP active site and, hence, explanation of the altered kinetic constants is difficult. Reorientation of the protein backbone due to residue substitution (K241A for example) could perhaps be a factor. A recent study indicates that such changes can occur [47]. Chemical modification of plant HRP with anthraquinone 2-carboxylic acid resulted in an $80 \%$ improvement in $k_{3} / \mathrm{K}_{\mathrm{m}}^{\prime}$ (the term used in that paper). Molecular dynamic simulations and experimental results indicated a molecular rearrangement: hydrophobic patches were redistributed, the access channel between Phe 68 and Phe 162 increased and the substrate binding area enlarged [47].

Elsewhere, single substitutions of non-active site residues affect catalytic activity. For example, Asn70 $\rightarrow$ Asp led to decreased oxidation of guaiacol [6], whilst Ser35 $\rightarrow$ Lys resulted in a 1.7-fold improvement in $\mathrm{V}_{\max } / \mathrm{K}^{\prime}{ }_{\mathrm{M}}$ ratio for $\mathrm{ABTS}$, possibly due to the introduction of a positive charge [5]. Such catalytic effects are not confined to HRP. Altered kinetic values for 2,4-dichlorophenol degradation were noted for a fungal lignin peroxidase following substitutions of numerous, mostly surface, sites [57]. A single Gly $\rightarrow$ Ser mutation far from the active site, but positioned in the entrance to the substrate binding site, benefited catalytic/ synthetic properties of gene-shuffled penicillin acylase [58]. 


\section{Conclusions.}

Suitable substitution of proximal helix G lysines 232 and 241, or glutamic acids 238 and 239, resulted in improved HRP catalytic properties with ABTS as reducing substrate. Modest gains in thermal stability, presumably by improved hydrogen bonding, hydrophobic rearrangements and/or space fill are also noted. Key stabilising roles for native Lys 174 and Glu 238 are shown. Double substitutions were neither additive nor synergistic, tending instead to match the lower stability characteristic. These rational mutations did not match the stability gains of chemically modified HRP; however, they do provide a platform for continued investigation of the stabilising determinants of this classical oxidoreductase.

\section{Acknowledgments}

We thank the Irish Research Council for Science, Engineering and Technology (Embark Initiative postgraduate scholarship to BJR) and Dublin City University (Postgraduate Accommodation Award to BJR and Albert College Award to CÓ'F) for financial support. We are also grateful to Prof FH Arnold and California Institute of Technology for the generous gift of a recombinant horseradish peroxidase plasmid. The National Centre for Sensors Research was established under the Higher Education Authority's Programme for Research in Third Level Institutions. 


\section{References.}

[1] Ryan BJ, Carolan N and O'Fágáin C. Horseradish and Soybean Peroxidases: Comparable Tools for Alternative Niches? Trends in Biotechnology 24 (2006) 355-363.

[2] Tanaka M., Ishimori K., Mukai M., Kitagawa T. and Morishima I. Catalytic activities and structural properties of horseradish peroxidase distal His42 to Glu or Gln mutant. Biochemistry, 36, (1997), 9889-9898.

[3] Savenkova M.I., Newmyer S.L. and De Montellano P.R.O. Rescue of His-42 to Ala horseradish peroxidase by a Phe-41 to His mutation - Engineering of a surrogate catalytic histidine. Journal of Biological Chemistry, 271, (1996), 24598-24603.

[4] Ozaki S. and Ortiz de Montellano P.R. Molecular engineering of horseradish peroxidase. Thioether sulfoxidation and styrene epoxidation by Phe-41 leucine and threonine mutants. J.Am.Chem.Soc., 117, (1995), 7056-7064.

[5] Tanaka M., Ishimori K. and Morishima I. Luminol activity of horseradish peroxidase mutants mimicking a proposed binding site for luminol in Arthromyces ramosus peroxidase. Biochemistry, 38, (1999), 10463-10473.

[6] Nagano S, Tanaka M, Watanabe Y. and Morishima I. Putative hydrogen bond network in the heme distal site of horseradish peroxidase. Biochem.Biophys.Res.Commun. 207, (1995), 417-423.

[7] Lipovsek D, Antipov E, Armstrong KA, Olsen MJ, Klibanov AM, Tidor B, Wittrup KD. Selection of horseradish peroxidase variants with enhanced enantioselectivity by yeast surface display. Chem Biol. 14, (2007), 1176-85.

[8] Pina DG, Shnyrova AV, Gavilanes F, Rodriguez A, Leal F, Roig MG, Sakharov IY, Zhadan GG, Villar E and Shnyrov VL. Thermally induced conformational changes in Horseradish Peroxidase. European Journal of Biochemistry 268, (2001), 120-126.

[9] Chattopadhyay K and Mazumdar S. Structural and conformational stability of Horseradish Peroxidase: effect of temperature and pH. Biochemistry 39, (2000) 263-270.

[10] Kaposi AD, Fidy J, Manas ES, Vanderkooi J.M and Wright WW. Horseradish Peroxidase monitored by infrared spectroscopy: effect of temperature, substrate and calcium. Biochimica et Biophysicia Acta 1435, 41-50.

[11] Tams JW and Welinder KG (1998) Glycosylation and thermodynamic versus kinetic stablilty of Horseradish Peroxidase. FEBS Letters 421, (1999) 234-236.

[12] Smeller L and Fidy J. The enzyme Horseradish Peroxidase is less compressible at higher pressures. Biophysical Journal 82 (2002), 426-436.

[13] Orlova MA, Kost OA, Gribkov VA, Gazaryan IG, Dubrovsky AV, Egorov VA. and Troshina NN. Enzyme activation and inactivation induced by low doses of irradiation. Applied Biochemistry and Biotechnology 88, (2000) 243-255. 
[14] Priori AM, Indiani C, de Sanctis G and Marini S. Anion and pH-linked conformational transition in Horseradish Peroxidase. Journal of Inorganic Biochemistry 79, (2000) 25-30.

[15] Moosavi-Movahedi AA and Nazari K. Denaturation of Horseradish Peroxidase with Urea and Guanidine Hydrochloride. International Journal of Biological Macromolecules 17, (1994) 43-47.

[16] Haque M E, Debnath D, Basak S and Chakrabarti A. Structural changes of Horseradish Peroxidase in presence of low concentrations of urea. European Journal of Biochemistry 259, (1999) 269-274

[17] Chakrabarti A and Basak S. Structural alterations of Horseradish Peroxidase in the presence of low concentrations of guandinium chloride. European Journal of Biochemistry 241, (1996) 462-467.

[18] Santucci R, Laurenti E, Sinibaldi F and Ferrari RP. Effect of dimethyl sulfoxide on the structure of the functional properties of Horseradish Peroxidase as observed by spectroscopy and cyclic voltammetry. Biochemica et Biophysica Acta 1596, (2002) 225-233.

[19] Machado MF and Saraiva JM. Thermal stability and activity regain of Horseradish Peroxidase in aqueous mixtures of imidazolium-based ionic liquids. Biotechnology Letters 27, (2005) 1233-1239.

[20] Morawski, B., S. Quan, and F.H. Arnold, Functional expression and stabilization of horseradish peroxidase by directed evolution in Saccharomyces cerevisiae. Biotechnol Bioeng, 76 (2001) 99-107.

[21] Liu JZ, Wang TL, Huang MT, Song HY, Weng LP and Ji LN. Increased thermal and organic solvent tolerance of modified horseradish peroxidase. Protein Engineering, Design and Selection, 19 (2006), 169-173.

[22] Gonera A, Mischnick P and Ukeda H. The application of O-aminopropyl amylose for the stabilisation of Horseradish Peroxidase via addition and crosslinking. Enzyme and Microbial Technology 34, (2004) 248-254.

[23] O'Brien AM, O'Fágáin C, Nielsen PF and Welinder KG. Location of Crosslinks in chemically stabilised Horseradish Peroxidase. Implications for design of crosslinks. Biotechnology and Bioengineering 76 (2001), 277-284.

[24] Miland E, Smyth MR. and O'Fágáin C. Modification of Horseradish Peroxidase with bifunctional N-hydroxysuccinimide esters: effects on molecular stability. Enzyme and Microbial Technology 19, (1996) 242-249.

[25] Ryan O, Smyth MR and O'Fágáin C. Thermostabilised chemical derivatives of Horseradish Peroxidase. Enzyme Microbial Technology 16, (1994) 501-505.

[26] Hassani L, Ranjbar B, Khajeh K, Naderi-Manesh H, Naderi-Manesh M and Sadeghi M. Horseradish peroxidase thermostabilization: The combinatorial effects of the surface modification and the polyols. Enzyme and Microbial Technology 38 (2006), 118-125. 
[27] O'Brien AM, Smith AT and O'Fágáin C. Effects of phthalic anhydride modification on Horseradish Peroxidase stability and activity. Biotechnology and Bioengineering, 81 (2003), 233-240.

[28] Liu JZ, Song HY, Weng LP and Ji LN. Increased thermostability and phenol removal efficiency by chemical modified horseradish peroxidase. Journal of Molecular Catalysis B 18, (2002) 225-232.

[29] Miland E, Smyth MR and O'Fágáin C. Increased thermal and solvent tolerance of acetylated Horseradish Peroxidase. Enzyme and Microbial Technology, 19, (1996) 63-67.

[30] Lin, Z., Thorsen T. and Arnold F.H. Functional expression of horseradish peroxidase in E. coli by directed evolution. Biotechnology Progress, 15 (1999) 457-471

[31] Grigorenko V, Chubar T, Kapeliuch Y, Borchers T, Spencer F and Egorov A New approaches for functional expression of recombinant horseradish peroxidase in Escherichia coli. Biocatalysis and Biotransformation 17, (1999) 359-379.

[32] Lei SP, Lin HC, Wang SS, Callaway J and Wilcox G. Characterisation of the Erwinia carotovora pelB gene and its product pectate lyase. Journal of Bacteriology, 169 (1987), 4379-4383.

[33] Sambrook J, Fritsch EF and Maniatis T (1989) Molecular Cloning- A laboratory Manual, 2 edn. Cold Spring Harbour Laboratory Press, Cold Spring Harbour, NY.

[34] Wang W and Malcom BA. Two-stage PCR protocol allowing introduction of multiple mutations, deletions and insertions using QuickChange ${ }^{\mathrm{TM}}$ site-directed mutagenesis. BioTechniques 26 (1999), 680-682.

[35] French C, Keshavarz-Moore E. and Ward J.M. Development of a simple method for the recovery of recombinant proteins from the E.coli periplasm. Enzyme Microbial Technology 19, (1996) 332-338.

[36] Smith AT, Sanders SA, Thorneley RNF, Burke JF and Bray RRC. Characterisation of a heme active-site mutant of horseradish peroxidase, 41-Phe-Val, with altered reactivity towards hydrogen peroxide and reducing substrates. European Journal of Biochemistry 207 (1992), 507-519.

[37] Chang BS, Park KH and Lund DB. Thermal inactivation kinetics of Horseradish Peroxidase. Journal of Food Science 53, (1988) 920-923.

[38] Khmelnitsky YL, Mozhaev VV, Belova AB, Sergeeva MV and Martinek K. Denaturation capacity: a new quantitative criterion for selection of organic solvents as reaction media in biocatalysis. European Journal of Biochemistry 198 (1991), 31-41.

[39] Dai, L. \& Klibanov, A.M. Striking activation of oxidative enzymes suspended in nonaqueous media. Proc. Nat. Acad. Sci. USA 96 (1999) 9475-9478

[40] Dunford HB (1999) Heme Peroxidases pp 1-23, 92-111, Wiley and Sons, NY. 
[41] Ugarova NN, Rozhkova GD. and Berezin IV. Chemical modification of the $\varepsilon$ aminogroups solflysine residues in HRP and its effect on the catalytic properties and thermostability. Biochemica et Biophysica Acta, 570, (1979) 31-42.

[42] Urrutigoity M, Baboulene M, Lattes A. Use of pyrocarbonate for chemical modification of histidine residues of horseradish peroxidase. Bioorganic Chemistry, 19, (1991) 66-76.

[43] Guex N and Peitsch MC. SWISS-MODEL and the Swiss-Pdb Viewer: An environment for comparative protein modelling. Electrophoresis 18, (1997) 2714-2723.

[44] Gajhede M, Schuller D, Henriksen A, Smith A and Poulos TL. Crystal structure of Horseradish Peroxidase C at $2.15 \AA$ resolution. Nature Structural Biology 4 (1997), 10321038.

[45] Howes BD, Feis A, Raimondi L, Indiani C and Smulevich G. The Critical Role of the Proximal Calcium Ion in the Structural Properties of Horseradish Peroxidase. Journal of Biological Chemistry 276 (2001), 40704-40711.

[46] Coates AI, Cook MP, Feezor R and Schuh MD. Dependence of Heme accessibility in Horseradish Peroxidase on $\mathrm{Ca}^{2+}$. Journal of Inorganic Biochemistry, 72, (1998) 63-69.

[47] Mogharrab N, Ghourchian H. and Amininasab M. Structural stabilisation and functional improvement of horseradish peroxidase upon modification of accessible lysines: experiments and simulation. Biophysical Journal, 92, (2007) 1192-1203.

[48] Batra R, Tyagi R and Gupta MN. Influence of immobilization on enzyme activity in aqeous-organic cosolvent mixtures. Biocatalysis and Biotransformation 15, (1997) 101-119.

[49] Gorman LAS. and Dordick JS. Organic-solvents strip water off enzymes. Biotechnology and Bioengineering 39 (1992), 392-397.

[50] Garcia D, Ortega F, Marty JL. Kinetics of inactivation of horseradish peroxidase: stabilizing effect of methoxypoly(ethylene glycol). Biotechnology and Applied Biochemistry 27, (1998) 49-54.

[51] Kwon KS, Kim J, Shin HS and Yu M-H. Single amino acid substitutions of $\alpha_{1}$ Antitrypsin that confer enhancement in thermal stability. Journal of Biological Chemistry 269, (1994) 9627-9631.

[52] Numata K, Hayashi-Iwasaki Y, Kawaguchi J, Sakurai M, Moriyama H, Tanaka N and Oshima T. Thermostabilisation of a chimeric enzyme by residue substitutions: four amino acid residues in loop regions are responsible for the thermostability of Thermus thermophilus isopropylmalate dehydrogenase. Biochimica et Biophysica Acta 1545, (2001) 174-183.

[53] Krowarsch D and Otlewski J. Amino acid substitutions at the fully exposed $\mathrm{P}_{1}$ site of bovine pancreatic trypsin inhibitor affect its stability. Protein Science 10, (2001) 715-724.

[54] Hakamada Y, Hatada Y, Ozawa T, Ozaki K, Kobayashi T and Ito S. Identification of thermostabilising residues in a Bacillus alkaline cellulase by construction of chimeras from 
mesophilic and thermostable enzymes and site directed mutagenesis. FEMS Microbiology Letters 195, (2001) 67-72.

[55] Ryan BJ and Ó'Fágáin C. Effects of single mutations on the stability of horseradish peroxidase to hydrogen peroxide. Biochimie 89: (2007) 1029-1032.

[56] Ignatenko, O.V., Gazaryan, I.G., Mareeva, E.A., Chubar, T.A., Fechina, V.A., Savitsky, P.A., Rojkova, A.M. and Tishkov, V.I. Catalytic properties of tryptophanless recombinant horseradish peroxidase. Biochemistry (Moscow), 64, (2000), 583-587.

[57] Ryu K, Hwang SY, Kim KH, Kang JH and Lee EK. Functionality improvement of fungal lignin peroxidase by DNA shuffling for 2,4-dichlorophenol degradability and $\mathrm{H}_{2} \mathrm{O}_{2}$ stability. J Biotechnol 133, (2008) 110-115.

[58] Jager SAW, Jekel PA, Janssen DB. Hybrid penicillin acylases with improved properties for synthesis of $\beta$-lactam antibiotics. Enzyme Microb Technol 40, (2007) 1335-1344. 


\section{Figure and Table Legends}

\section{Figure 1: Schematic representation of pQE60_PelB_HRP_His, termed pBR_I.}

The pQE60 vector contains the PelB leader and HRP gene flanked by the initiation codon at the 5' end and by the poly His purification tag and stop codon at the 3' end of the multiple cloning site. (Amp,

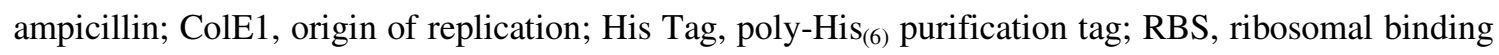
site), Image generated using Redasoft Visual Cloning 3.2 (www.rebase.neb.com).

Figure 2: Thermal profile of Lysine 241 mutants.

$\Delta \mathrm{K} 241 \mathrm{~A}, \square \mathrm{K} 241 \mathrm{E}, \diamond \mathrm{K} 241 \mathrm{~N}, \mathrm{x}$ K232F, $\circ \mathrm{WT}$. For details, see Methods.

Figure 3: Partial native and lysine substituted hydrogen-bond network surrounding the proximal calcium.

Part A displays the hydrogen bond network around the heme group and proximal calcium ion. The hepta co-ordinated calcium ion (Thr171 [two bonds], Asp 222, Thr 225 [two bonds], Ile 228 and Asp230; [45]) is represented as a grey sphere. Lysine 232 is coloured black, whilst H-bonds are dashed grey lines. Part B illustrates likely novel H-bonds developed by the K232N mutation carried out in this study. Images generated in DeepView [43].

Table 1: Characteristics of Recombinant HRP Variants with Substitutions in the Helix G Region.

Apparent $V_{\mathrm{m}} / E$ and $K_{\mathrm{m}}^{\prime}$ values, first-order rate constant $k$ and half-life $\left(t_{1 / 2}\right)$ at $50^{\circ} \mathrm{C}$, and calculated solvent $\mathrm{C}_{50}$ for each Lysine and Glutamic Acid mutant. ABTS was the reducing substrate for kinetic analysis. $k$-values, apparent $V_{\mathrm{m}} / E$ and $K_{\mathrm{m}}^{\prime}$ values, including standard errors (SE), were calculated using the Enzfitter ${ }^{\mathrm{TM}}$ software package (Version 1.05. Cambridge UK: Biosoft Ltd.; 1987). Values are the mean of three independent experiments in all cases. *For all solvents studied, standard errors were < 5\%. N/D, not determined. (Thermal inactivation of K241N and K241E did not fit a first-order model.) 
Table 1: Characteristics of Recombinant HRP Variants with Substitutions in the Helix G Region.

Apparent $V_{\mathrm{m}} / E$ and $K_{\mathrm{m}}^{\prime}$ values, first-order rate constant $k$ and half-life $\left(t_{1 / 2}\right)$ at $50^{\circ} \mathrm{C}$, and calculated solvent $\mathrm{C}_{50}$ for each Lysine and Glutamic Acid mutant. ABTS was the reducing substrate for kinetic analysis. $k$-values, apparent $V_{\mathrm{m}} / E$ and $K_{\mathrm{m}}$ values, including standard errors (SE), were calculated using the Enzfitter ${ }^{\mathrm{TM}}$ software package (Version 1.05. Cambridge UK: Biosoft Ltd.; 1987). Values are the mean of three independent experiments in all cases. *For all solvents studied, standard errors were < 5\%. N/D, not determined. (Thermal inactivation of K241N and K241E did not fit a first-order model.)

\begin{tabular}{|c|c|c|c|c|c|c|c|c|c|c|}
\hline \multirow{3}{*}{$\begin{array}{c}\text { Mutant } \\
\text { Wildtype }\end{array}$} & \multicolumn{4}{|c|}{ ABTS Steady-State Kinetics } & \multicolumn{3}{|c|}{ Thermal Stability } & \multicolumn{3}{|c|}{ Solvent Stability* } \\
\hline & \multicolumn{2}{|c|}{$\begin{array}{c}\boldsymbol{V}_{\mathbf{m}} / \boldsymbol{E} \\
\left(\mathrm{s}^{-1}\right)\end{array}$} & \multicolumn{2}{|c|}{$\begin{array}{l}\mathbf{K}_{\mathbf{m}}^{\prime} \\
\mathrm{mM}\end{array}$} & \multirow{2}{*}{$\begin{array}{c}\boldsymbol{k} \\
\left(\mathrm{min}^{-1}\right) \\
0.056\end{array}$} & \multirow{2}{*}{$\begin{array}{c}\text { SE } \\
\left(\min ^{-1}\right) \\
\pm 0.003\end{array}$} & \multirow{2}{*}{$\begin{array}{c}\mathbf{t}_{1 / 2} \\
(\min )\end{array}$} & \multirow{2}{*}{$\begin{array}{c}\text { DMSO } \\
\mathrm{C}_{50} \\
35\end{array}$} & \multirow{2}{*}{$\begin{array}{c}\text { МeOH } \\
\mathrm{C}_{50} \\
53\end{array}$} & \multirow{2}{*}{$\begin{array}{c}\text { DMF } \\
\text { C }_{50} \\
14\end{array}$} \\
\hline & 482 & \pm 12 & 0.093 & \pm 0.013 & & & & & & \\
\hline K174N & 624 & \pm 14 & 0.142 & \pm 0.013 & 0.087 & \pm 0.009 & 8 & 42 & 45 & 15 \\
\hline K174E & 354 & \pm 19 & 0.142 & \pm 0.032 & 0.102 & \pm 0.003 & 7 & 20 & 55 & 13 \\
\hline $\mathrm{K} 174 \mathrm{~A}$ & 954 & \pm 12 & 0.096 & \pm 0.007 & 0.068 & \pm 0.005 & 10 & 30 & 35 & 19 \\
\hline $\mathrm{K} 232 \mathrm{~N}$ & 1849 & \pm 103 & 0.168 & \pm 0.038 & 0.031 & \pm 0.004 & 22 & 45 & 45 & 48 \\
\hline $\mathrm{K} 232 \mathrm{E}$ & 467 & \pm 18 & 0.131 & \pm 0.022 & 0.060 & \pm 0.005 & 12 & 38 & 33 & 15 \\
\hline $\mathrm{K} 232 \mathrm{~A}$ & 433 & \pm 10 & 0.118 & \pm 0.013 & 0.092 & \pm 0.007 & 8 & 40 & 35 & 21 \\
\hline $\mathrm{K} 232 \mathrm{~F}$ & 983 & \pm 38 & 0.126 & \pm 0.034 & 0.029 & \pm 0.002 & 24 & 55 & 67 & 60 \\
\hline E238Q & 1298 & \pm 63 & 0.022 & \pm 0.034 & 0.092 & \pm 0.01 & 8 & 30 & 45 & 10 \\
\hline E239N & 526 & \pm 23 & 0.013 & \pm 0.034 & 0.057 & \pm 0.006 & 12 & 37 & 53 & 13 \\
\hline $\mathrm{K} 241 \mathrm{~N}$ & 1141 & \pm 41 & 0.092 & \pm 0.017 & $\mathrm{~N} / \mathrm{D}$ & -- & $\mathrm{N} / \mathrm{D}$ & 38 & 55 & 18 \\
\hline $\mathrm{K} 241 \mathrm{E}$ & 978 & \pm 64 & 0.062 & \pm 0.018 & N/D & -- & $\mathrm{N} / \mathrm{D}$ & 30 & 38 & 15 \\
\hline $\mathrm{K} 241 \mathrm{~A}$ & 2410 & \pm 157 & 0.320 & \pm 0.075 & 0.113 & \pm 0.013 & 6 & 35 & 38 & 18 \\
\hline $\mathrm{K} 241 \mathrm{~F}$ & 880 & \pm 20 & 0.308 & \pm 0.038 & 0.061 & \pm 0.005 & 11 & 34 & 43 & 20 \\
\hline $\mathrm{K} 232 \mathrm{Q} / \mathrm{K} 241 \mathrm{Q}$ & 1549 & \pm 61 & 0.114 & \pm 0.036 & 0.045 & \pm 0.004 & 15 & 32 & 48 & 9 \\
\hline $\mathrm{K} 232 \mathrm{~N} / \mathrm{K} 241 \mathrm{~N}$ & 378 & \pm 30 & 0.197 & \pm 0.068 & 0.036 & \pm 0.004 & 19 & 35 & 48 & 20 \\
\hline $\mathrm{K} 232 \mathrm{~F} / \mathrm{K} 241 \mathrm{~N}$ & 1008 & \pm 39 & 0.114 & \pm 0.018 & 0.025 & \pm 0.002 & 27 & 62 & 65 & 62 \\
\hline
\end{tabular}




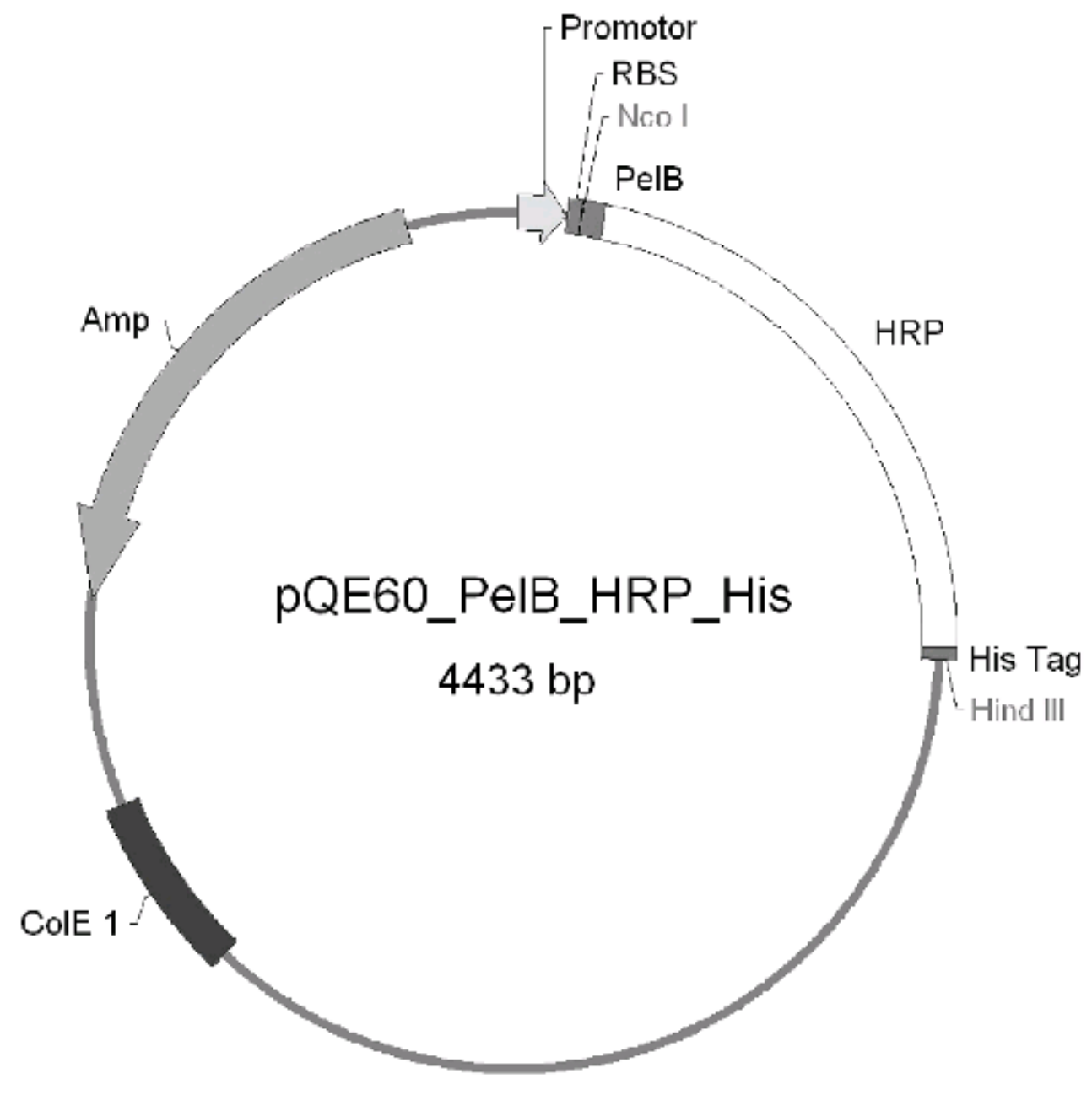




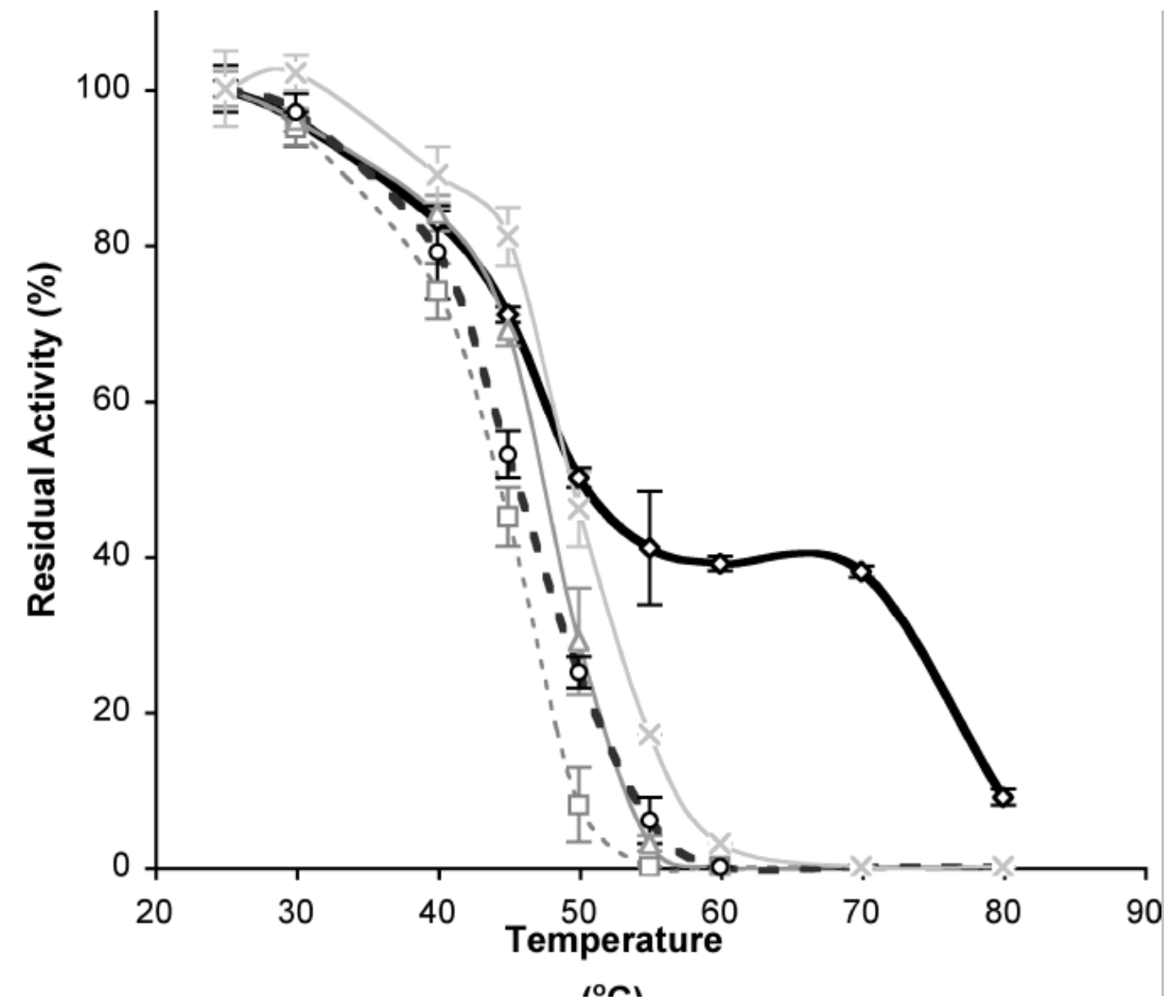




$$
\text { 资 }
$$

